\title{
Geringe Literalität und politische Grundbildung: Die Bedeutung schriftsprachlicher Fähigkeiten für die Selbsteinschätzung politischer Grundkompetenzen
}

\author{
Gregor Dutz (D)
}

Eingegangen: 13. August 2020 / Überarbeitet: 5. Oktober 2020 / Angenommen: 23. Oktober 2020 /

Online publiziert: 18 . November 2020

(C) Der/die Autor(en) 2020

Zusammenfassung Politische Grundbildung hat sich inzwischen als ein inhaltlicher Pfeiler in der Diskussion um Grundbildung etabliert, wobei über die politikbezogenen Grundkompetenzen gering literalisierter Erwachsener bisher wenig bekannt ist. Basierend auf den Ergebnissen der Studie LEO 2018 und mithilfe logistischer Regressionsmodelle wird die These überprüft, dass gering literalisierte Erwachsene die eigenen politikbezogenen Kompetenzen als eher gering einschätzen und sich so vom politischen Feld ausschließen. Dabei zeigt sich, dass auch bei der Kontrolle verschiedener soziökonomischer Faktoren gering literalisierte Erwachsene mit einer höheren Wahrscheinlichkeit die eigenen Kompetenzen als eher niedrig einschätzen.

Schlüsselwörter Alphabetisierung · Politische Bildung · Politische Grundbildung • Partizipation · Logistische Regression

\section{Low literacy and basic civic education: the relevance of literacy to self- assessed basic political competences}

\begin{abstract}
Basic civic education is discussed as a substantial pillar in the discussion on basic education, although little is known about the basic civic skills of adults with low literacy skills. In this article based on the results of LEO 2018 and with logistic regression models, the thesis is examined that low-literate adults assess their own civic skills as rather low and thus exclude themselves from the political field. It is shown that even when controlling for various socio-economic factors, low-literate adults are more likely to assess their own competences as rather low.
\end{abstract}

Keywords Literacy - Civic education - Basic civic education · Participation · Logistic regression 


\section{Einleitung}

Die politische Grundbildung hat sich inzwischen als ein Teilbereich von Grundbildung etabliert (Duncker-Euringer 2017; Mania und Tröster 2018). Dabei ist sie inhaltlich vergleichsweise wenig definiert (Korfkamp 2016) und bestimmt sich mehr über ihre Adressatinnen und Adressaten sowie ihre Ziele (vgl. Zeuner 2017). Hierbei werden besonders häufig Menschen aus Milieus mit geringen literalen Fähigkeiten und einem Habitus, der sowohl die Teilnahme an politischer Bildung als auch an politischer Partizipation negativ beeinflusst, als Adressatinnen und Adressaten angesprochen (Bremer 2010; Koppel und Schieferdecker 2019).

Über die tatsächliche politische Partizipation und die politikbezogenen Kompetenzen von Erwachsenen mit geringer Literalität ist bisher wenig bekannt. Vorhandene Erkenntnisse beziehen sich meist auf Teilnehmende von Grundbildungskursen (Bremer und Pape 2017; Pape 2011) oder die Auswertung einzelner Fragen aus quantitativen Kompetenzstudien (Dutz und Heilmann 2020; Grotlüschen 2016). Weiterführende Erkenntnisse sind hier von großer Bedeutung für die Erwachsenenbildung. Zwar entstehen nicht zuletzt im Internet neue Formate und Möglichkeiten politischer Beteiligung (van Deth 2009). Diese können potenziell auch die Teilhabewege derjenigen erweitern, die bisher weniger am politischen Geschehen partizipierten. Dennoch bleibt die Teilnahme am politischen Diskurs weiterhin voraussetzungsreich.

Neben dem politischen Sachwissen erfordern komplexe Sachverhalte und die überwiegend schriftlichen Diskussionsformen, etwa in sozialen Netzwerken oder auf den Webseiten von Zeitungen, das Beherrschen der (deutschen) Bildungssprache, sowohl in schriftlicher als auch in nicht-schriftlicher Form. Weiterhin sind Medienkompetenz und Urteilsfähigkeit erforderlich, um falsche oder verfälschende Nachrichten (fake news) zu entdecken oder populistische Meinungsäußerungen zu erkennen (Grimm et al. 2019).

Es wird auch diskutiert, ob bei gering literalisierten Erwachsenen die Gefahr von Entsolidarisierung und die Hinwendung zu populistischen und rechtsextremen Parteien bestehe, hervorgerufen durch ein niedriges politisches Selbstwirksamkeitsgefühl und niedriges soziales Vertrauen (Grotlüschen 2016). In diesem Kontext ist auch festzustellen, dass nicht jede Art der politischen Partizipation aus demokratischer Perspektive als positiv zu bewerten ist (van Deth 2013b).

In quantitativen Studien wurden die Lese- und Schreibkompetenz und politikbezogene Kompetenzen bisher nicht gemeinsam erhoben. Die Daten der Studie LEO 2018 - Leben mit geringer Literalität (LEO 2018) erlauben eine Auswertung hinsichtlich der Frage, ob gering literalisierte Erwachsene ihre politikbezogenen Kompetenzen - unter Berücksichtigung verschiedener sozioökonomischer Merkmale geringer einstufen als höher literalisierte Erwachsene.

\section{Forschungslage und theoretischer Rahmen}

In der Diskussion um politische Grundbildung spielen gering literalisierte Erwachsene eine zentrale Rolle, da sie zum einen verschiedene Eigenschaften mit ressourcen- 
schwachen Personengruppen teilen, die in der Partizipationsforschung als wenig partizipierend identifiziert werden (van Deth 2013a). Ein niedriger Bildungsstand oder Erwerbslosigkeit sind bei gering literalisierten Erwachsenen häufiger anzutreffen als in der Gesamtbevölkerung. In der Gruppe der gering literalisierten Erwachsenen findet sich auch ein vergleichsweise hoher Anteil von Menschen mit Migrationshintergrund, die seltener politisch partizipieren (Wiedemann 2006). Zum anderen wird auch angenommen, dass ihnen die für eine umfassende politische Partizipation notwendigen Kompetenzen, sowohl schriftsprachlicher als auch politikbezogener Art, zumindest teilweise fehlen (Korfkamp 2016; Zeuner 2017).

Einen systematischen Überblick über den Zusammenhang zwischen (geringer) Literalität und gesellschaftlicher Teilhabe bieten Koppel und Schieferdecker (2019): Basierend auf der Analyse der materiellen, kulturellen, sozialen und politischen Dimension kommen die Autorinnen und Autoren zu dem Schluss, dass

gerade für Menschen mit erkennbar begrenzten Verwirklichungschancen [dazu gehören gering literalisierte Erwachsene, Anm. d. Verf.] die Gefahr [besteht], sich bei gesellschaftlichen Veränderungsprozessen weniger stark einbringen zu können. (Koppel und Schieferdecker 2019, S. 170)

Quantitative Studien haben den Zusammenhang zwischen Literalität und politischer Partizipation sowie politikbezogenen Kompetenzen Erwachsener nur selten zum Thema (vgl. Grotlüschen 2016, S. 184). Das Programme for the International Assessment of Adult Competencies (PIAAC), eine international vergleichende Studie der OECD, erfasst im Sinne eines engen Verständnisses von Politik die externe politische Selbstwirksamkeitserwartung, soziales Vertrauen und die Häufigkeit freiwilligen Engagements. Die Analysen dieser Fragen zeigen, dass gering literalisierte Erwachsene eine niedrigere politische Selbstwirksamkeitserwartung und weniger soziales Vertrauen besitzen sowie sich seltener freiwillig engagieren als höher literalisierte Erwachsene (Grotlüschen 2016; Grotlüschen et al. 2016).

Die Daten der deutschen längsschnittlichen PIAAC-Fortführung (PIAAC-L) ergeben, dass Erwachsene mit einer niedrigen Schreibkompetenz seltener bestimmte politische Praktiken ausüben. Ein eindeutiger Zusammenhang zwischen Literalität und politischen Praktiken konnte jedoch in multivariaten Auswertungen nicht gezeigt werden, wichtiger war der Einfluss von Bildungsstand und politischer Selbstwirksamkeitserwartung (Dutz und Heilmann 2020).

Bourdieu verweist auf zwei Dimensionen politischer Kompetenz. Zum einen Kompetenz „,im Sinne sachlicher Fähigkeit“, zum anderen ,im Sinne von Rechtsfähigkeit“" (Bourdieu 1987, S. 623), also einem Gefühl (un-)berechtigt zu sein, sich politisch äußern oder politisch handeln zu können (vgl. Müller 2016, S. 262). Diejenigen, die kompetent in beiderlei Sinne sind, erfüllen die „Zugangsvoraussetzungen“ (Bourdieu 2013, S. 99) zum politischen Feld. Diejenigen, die diese nicht erfüllen, bleiben davon ausgeschlossen.

In diesem Kontext untersuchen Bremer und Pape (2017) den Zusammenhang zwischen Literalität und politischer Partizipation. Sie erkennen bei gering literalisierten Erwachsener ein geringes Selbstvertrauen in diese von Bourdieu genannten Dimensionen politischer Kompetenz. Gering literalisierte Erwachsene schreiben sich selbst ein eher geringes Interesse an Politik zu, fühlen sich ausgeschlossen und 
schätzen ihre eigenen (schriftsprachlichen) Fähigkeiten als ungenügend ein, um am politischen Diskurs teilzuhaben (Bremer und Pape 2017; Pape 2011). Bremer und Pape (2017) konstatieren, dass einfache politikbezogene Handlungen - das Lesen von Wahlunterlagen wird beispielhaft genannt - für gering literalisierte Erwachsene kein großes Problem darstellen würden, politische Partizipation aber ,,milieuspezifischen Mustern“ folge (Bremer und Pape 2017, S. 68). Hier zeigt sich auch ein Zusammenhang mit dem Konzept legitimer Sprache (vgl. Grotlüschen et al. 2009): So sei die „Nicht-Beherrschung des, legitimen“ politischen Stils eng verknüpft (...) mit der Nicht-Beherrschung der legitimen Schriftsprache, was zu Mechanismen des Selbst- und Fremdausschlusses aus dem ,politischen Spiel ' führt" (Bremer und Pape 2017, S. 68). Fehlende schriftsprachliche Kenntnisse können also auf diesem Wege zu geringerer politischer Partizipation führen. Die Autorinnen und Autoren weisen auch darauf hin, dass politisches Interesse durchaus vorhanden sei, auch wenn es nicht immer als solches (an-)erkannt werde.

Die LEO-Studie 2018 untersucht die gesellschaftliche Teilhabe von Erwachsenen in verschiedenen Lebensbereichen in Deutschland (Grotlüschen et al. 2020). Sie erfasst die Lese- und Schreibkompetenz der deutschen Bevölkerung und ermöglicht durch die detaillierte Betrachtung verschiedener Alltagsbereiche die Untersuchung möglicher Teilhabechancen und -ausschlüsse. Neben verschiedenen schriftlichen und nicht-schriftlichen politikbezogenen Praktiken werden auch Selbsteinschätzungen politikbezogener Grundkompetenzen erhoben. Hinsichtlich vieler Praktiken, etwa des Wählens oder des Lesens einer Zeitung, belegen deskriptive Auswertungen, dass gering literalisierte Erwachsene diese seltener ausüben als der Bevölkerungsdurchschnitt (Dutz und Grotlüschen 2020). Jedoch zeigt sich auch hier, dass gering literalisierte Erwachsene nicht pauschal desinteressiert sind: Eine große Mehrheit der gering literalisierten Erwachsenen bekommt täglich mit, was in Deutschland und der Welt passiert und schaut kaum seltener als höher literalisierte Erwachsene Nachrichtensendungen.

Betrachtet man aber nicht nur die politikbezogenen Alltagspraktiken, sondern auch die selbsteingeschätzten politikbezogenen Grundkompetenzen, werden deutliche Unterschiede zwischen gering und höher literalisierten Erwachsenen sichtbar. Beispielhaft sei hier eine Frage aus LEO 2018 herausgegriffen: Wie einfach/ schwierig ist es für Sie zu beurteilen, ob eine bestimmte politische Partei das vertritt, was Sie wichtig finden? Von den gering literalisierten Erwachsenen antworten auf diese Frage 43,8\%, dass sie dies (eher) schwierig fänden; bei den höher literalisierten Erwachsenen beträgt dieser Anteil nur 22,5\% (Dutz und Grotlüschen 2020, S. 315).

Diese Daten erlauben somit erstmals einen repräsentativen Blick auf politikbezogene Grundkompetenzen gering literalisierter Erwachsener und damit auch multivariate Analysen. Die Ergebnisse aus diesen Analysen können ergänzend zu Erkenntnissen aus qualitativen Studien über die Teilnehmenden von Grundbildungskursen hinzugenommen werden. Zusammengenommen helfen diese Erkenntnisse dabei, ein vollständigeres Bild von möglichen Teilhabeausschlüssen und Lernbedarfen in diesem Bereich zu zeichnen. Einschränkend soll erwähnt werden, dass selbsteingeschätzte Grundkompetenzen nicht zwingend den in einem Test gemessenen Kompetenzen entsprechen müssen (Edele et al. 2015). Bei der Interpretation der 
Ergebnisse soll daher das oben erwähnte Kompetenzverständnis von Pierre Bourdieu Beachtung finden. Konkret für diese Untersuchung bedeutet dies, dass die Selbsteinschätzung der politischen Kompetenzen gering literalisierter Erwachsener zum einen durch fehlende schriftsprachliche Fähigkeiten (etwa beim Lesen oder Schreiben komplexer politischer Texte) beeinflusst werden kann, zum anderen durch das Gefühl fehlender Legitimation, politisch Handeln zu dürfen.

Basierend auf der Forschungslage soll die Hypothese untersucht werden, dass gering literalisierte Erwachsene aus diesen Gründen niedrigere subjektive, politikbezogene Grundkompetenzen besitzen als höher literalisierte Erwachsene; dabei werden verschiedene sozioökonomische Merkmale berücksichtigt.

Die erkenntnisleitende Forschungsfrage für diesen Beitrag lautet daher: Gibt es einen Zusammenhang zwischen schriftsprachlichen Fähigkeiten (Literalität) und den selbsteingeschätzten politikbezogenen Grundkompetenzen, unter Berücksichtigung verschiedener sozioökonomischer Merkmale?

\section{Daten und Methode}

Als Datengrundlage für die hier durchgeführte Sekundäranalyse dienen die im Rahmen der Studie LEO 2018 erhobenen Daten über 7192 Befragte (Grotlüschen et al. 2020). Die Zufallsstichprobe der Studie ist repräsentativ für die deutschsprachige Wohnbevölkerung in Privathaushalten im Alter von 18 bis 64 Jahren in Deutschland.

Die inhaltlichen Schwerpunkte von LEO 2018 liegen zum einen in der Erfassung der Lese- und Schreibkompetenz der deutschsprachigen Bevölkerung - insbesondere im unteren Kompetenzbereich - zum anderen widmet sich die Studie den schriftlichen und nicht-schriftlichen Praktiken und Grundkompetenzen in verschiedenen Lebensbereichen.

Die Studie wurde als Computer-unterstützte, persönliche Befragung (CAPI) durchgeführt, der Kompetenztest wurde von den Befragten mit Stift und Papier bearbeitet. Eine ausführliche Beschreibung der Studie und die Darstellung der zentralen Studienergebnisse finden sich bei Grotlüschen und Buddeberg (2020).

Durch die Erfassung von digitalen, finanzbezogenen, gesundheitsbezogenen und politikbezogenen Praktiken und Grundkompetenzen sollen durch geringe Literalität bedingten Gefahren des Teilhabeausschlusses in diesen Bereichen untersucht werden. In geringerem Umfang wurden auch Praktiken aus den Bereichen Arbeit, Familie, Weiterbildung, Migration und Mehrsprachigkeit erhoben, ergänzt durch soziodemografische Merkmale, etwa zum Bildungsstand oder dem Erwerbsstatus.

In diesem Beitrag soll entsprechend der oben formulierten Forschungsfrage der Einfluss von Literalität auf die selbsteingeschätzten politikbezogenen Grundkompetenzen, unter Kontrolle verschiedener soziodemografische Variablen, untersucht werden. Für die Auswertung sollen logistische Regressionen mit einer binären abhängigen Variablen durchgeführt werden. Das Vorgehen und die verwendeten Variablen werden im Folgenden erläutert. 


\subsection{Abhängige Variablen}

Die politikbezogenen Grundkompetenzen werden in LEO 2018 durch insgesamt sechs Fragen erfasst. Diese Grundkompetenzen werden nicht getestet, sondern basieren auf der Selbstauskunft der Befragten. Drei dieser Fragen lassen sich den funktional-pragmatischen Kompetenzen, drei weitere den kritisch-hinterfragenden Kompetenzen zuordnen. Als funktional-pragmatische Kompetenzen werden in LEO 2018 Kompetenzen verstanden, die auf die erfolgreiche Ausführung einer Handlung abzielen (vgl. Klieme und Leutner 2006); als kritisch-hinterfragende Kompetenzen werden Kompetenzen verstanden, die das Hinterfragen und Beurteilen von Problemen zum Gegenstand haben (vgl. Negt 1990; ausführlicher zum Kompetenzverständnis in LEO 2018 siehe Grotlüschen et al. 2020, S. 18f.).

Die Fragen zu funktional-pragmatischen politikgezogenen Grundkompetenzen lauten:

$1 a$ Trauen Sie sich zu, in einer Auseinandersetzung mit einem Amt oder einer Behörde die eigene Meinung zu vertreten?

1b Trauen Sie sich zu, wichtige politische Fragen gut zu verstehen und einzuschätzen?

1c Trauen Sie sich zu, an einem Gespräch über politische Fragen aktiv teilzunehmen?

Die Antwortmöglichkeiten für diese Fragen sind „ohne Schwierigkeiten“, „mit gewissen Schwierigkeiten“, „mit großen Schwierigkeiten“ und ,gar nicht“. Für die logistische Regression werden die entsprechenden Variablen dichotomisiert, indem jeweils die Kategorien „ohne Schwierigkeiten“ und „mit gewissen Schwierigkeiten“ sowie die Kategorien „mit großen Schwierigkeiten“ und „gar nicht“ zusammengefasst werden. Die so gebildete Variable hat die Ausprägung ,1" für die Kategorien „ohne Schwierigkeiten/mit gewissen Schwierigkeiten“, die Ausprägung „0“ für die Kategorien „mit großen Schwierigkeiten/gar nicht“.

Die Fragen zu den kritisch-hinterfragenden politikbezogenen Grundkompetenzen lauten:

2a Wie einfach/schwierig ist es für Sie zu beurteilen, ob Sie sich an einer Unterschriftenliste oder -aktion beteiligen wollen?

2b Wie einfach/schwierig ist es für Sie, an einer Demonstration teilzunehmen?

2c Wie einfach/schwierig ist es für Sie zu beurteilen, ob eine bestimmte politische Partei das vertritt, was Sie wichtig finden?

Hier lauten die Antwortmöglichkeiten ,einfach“, ,eher einfach“, ,,eher schwierig“ und „schwierig“. Zusammengefasst werden jeweils die Kategorien ,einfach“ und „eher einfach“" sowie ,eher schwierig“ und ,schwierig“. Die so gebildete Variable hat die Ausprägung „, “ für die Kategorien ,einfach/eher einfach“, die Ausprägung „,0“ für die Kategorien ,eher schwierig/schwierig“. 


\subsection{Unabhängige Variablen}

Zur Überprüfung der Hypothese, ob es einen Zusammenhang zwischen Literalität und den selbsteingeschätzten politikbezogenen Kompetenzen gibt, fließt Literalität als zentrale erklärende Variable in die Regressionsmodelle ein. Als weitere unabhängige Variablen gehen in die Modelle eine Reihe weiterer Kontrollvariablen ein.

\subsubsection{Literalität}

Die Lese- und Schreibkompetenz (Literalität) in der deutschen Sprache wird in der Studie LEO 2018 mithilfe eines Kompetenztests (Assessment) gemessen, welcher zu großen Teilen auf der ersten LEO-Studie 2010 basiert (Grotlüschen und Riekmann 2012). Im Kompetenztest bearbeiteten die Befragten eine Reihe von Aufgaben (Testitems). Die ausgefüllten Testhefte wurden im Anschluss von geschulten Editierkräften nach festgelegten Regeln ausgewertet und in „richtig“ bzw. ,falsch“ codiert. Auf Grundlage dieser Antworten wurden die Test-Items mithilfe der Item-ResponseTheorie skaliert. Als Schätzer für die Personenfähigkeiten wurden anschließend mit einer latenten Regression und einem Hintergrundmodell für jede befragte Person zehn sogenannte plausible Werte ermittelt, die die Personenfähigkeit auf der LEOSkala verzeichneten. Das genaue Vorgehen wird bei Dutz und Hartig (2020) beschrieben.

Die Konzeption der Test-Items erlaubt es, die berechneten Personenfähigkeiten kriterienorientiert zu interpretieren (Hartig et al. 2012). Dies geschieht auf Grundlage der sogenannten Alpha-Levels, die die LEO-Skala in verschiedene Kompetenzlevels einteilt. Die Alpha-Levels 1-3 beschreiben dabei den Bereich geringer Literalität. Dies bedeutet, dass eine Person mit einer Lese- und Schreibkompetenz auf einem dieser Levels die deutsche Schriftsprache maximal auf der Ebene einfacher Sätze beherrscht (Grotlüschen et al. 2020, S. 15).

Für die hier dargestellten Auswertungen werden die Alpha-Levels in eine dichotomisierte Variable recodiert. Eine Gruppe bilden dabei die gering literalisierten Erwachsenen (Alpha-Levels 1-3), die zweite Gruppe setzt sich aus Erwachsenen mit einer höheren Lese- und Schreibkompetenz zusammen (Alpha-Level 4 und darüber). Die statistische Referenzgruppe bilden diejenigen auf Alpha-Level 4 und darüber.

\subsubsection{Kontrollvariablen}

Als Kontrollvariablen werden die folgenden Variablen aufgenommen, da für diese bekannt ist, dass sie im Zusammenhang mit politikbezogener Partizipation oder Grundkompetenz stehen (Fuchs 2006; van Deth 2013a; Verba et al. 1995; Wiedemann 2006). In Klammern wird jeweils die Referenzkategorie der Dummy-Variablen angegeben:

- Geschlecht (weiblich)

- Alter in Altersgruppen (35-44 Jahre)

- Schulabschluss (hoch)

- höchster Schulabschluss der Eltern (hoch) 
- Beschäftigungsstatus (erwerbstätig)

- Auskommen mit dem Haushaltseinkommen (eher gut und gut)

- Herkunftssprache (deutsch)

- Staatsbürgerschaft (deutsch)

\subsection{Regressionsmodelle}

Zunächst wird für jede der oben dargestellten Kompetenzfragen eine einfache binäre logistische Regressionsanalyse durchgeführt, um den Zusammenhang zwischen Literalität und den subjektiven politischen Grundkompetenzen zu untersuchen. Anschließend werden die weiteren abhängigen Variablen zur logistischen Regression hinzugefügt. So kann untersucht werden, ob ein Zusammenhang zwischen Literalität und Grundkompetenzen auch bestehen bleibt, wenn auf weitere Eigenschaften der Befragten hin kontrolliert wird.

Dabei sollen für beide Kompetenzbereiche die gleichen abhängigen Variablen gewählt werden. Wie oben dargestellt, ist die Forschungslage zu quantitativen Untersuchungen über selbsteingeschätzte politikbezogene Grundkompetenzen eher dünn, daher basiert die Auswahl der Kontrollvariablen stark auf Ergebnisse aus der Partizipationsforschung. Die hier durchgeführten Analysen haben somit auch einen explorativen Charakter und ermöglichen den Vergleich der Ergebnisse über die beiden Kompetenzbereiche hinweg.

Bei der Auswertung kommt die Statistiksoftware R mit den Paketen survey (Lumley 2018b) und mitools (Lumley 2018a) zum Einsatz.

\subsection{Sample}

Das Sample für die durchgeführten Analysen umfasst prinzipiell alle Antworten der in LEO 2018 erfassten 7192 Befragten. Da nicht alle Fragen von allen Befragten beantwortet wurden, kann die tatsächliche Zahl der in die Regressionen einfließenden Fälle geringer sein.

Dutz und Grotlüschen (2020, S. 313) stellen in der deskriptiven Analyse der Kompetenzfragen fest, dass insbesondere bei den Fragen zu den kritisch-hinterfragenden politikbezogenen Grundkompetenzen ein vergleichsweise großer Anteil der Befragten mit ,weiß nicht“ geantwortet hat und deshalb aus dem Sample herausfällt. Bei den gering literalisierten Erwachsenen ist dieser Anteil mit jeweils knapp über $10 \%$ besonders hoch. Die Beantwortung der Fragen fällt den gering literalisierten Erwachsenen also besonders schwer und es kann vermutet werden, dass ein Teil derjenigen, die ihre Kompetenzen als besonders niedrig einschätzen würden, daher nicht in die Regressionsanalyse einfließen.

\section{Ergebnisse}

In diesem Abschnitt sollen die Ergebnisse der Regressionsanalysen dargestellt werden. Zunächst werden die funktional-pragmatischen, anschließend die kritisch-hin- 
terfragenden politikbezogenen Grundkompetenzen betrachtet. Die Darstellung der Regressionskoeffizienten in den Tabellen erfolgt dabei als odds ratio. Dabei kann ein Wert unter 1,0 als eine niedrigere Wahrscheinlichkeit im Vergleich zur Referenzgruppe interpretiert werden, auf die gestellten Fragen „ohne Schwierigkeiten/mit gewissen Schwierigkeiten“ bzw. „einfach/eher einfach“ zu antworten. Umgekehrt zeigt ein Wert von über 1,0 an, dass im Mittel eine höhere Wahrscheinlichkeit besteht, so zu antworten (zur Interpretation der Regressionskoeffizienten siehe auch Best und Wolf 2010). Vereinfacht gesagt kann ein Wert unter 1,0 also als eine im Durchschnitt niedriger eingeschätzte politikbezogene Grundkompetenz, ein Wert über 1,0 als eine im Durchschnitt höher eingeschätzte politikbezogene Grundkompetenz der jeweiligen Gruppe interpretiert werden. In Klammern wird zusätzlich das Konfidenzintervall auf dem $95 \%$-Niveau angegeben.

\subsection{Funktional-pragmatische Grundkompetenzen}

Tab. 1 stellt das Ergebnis der logistischen Regressionsanalyse der funktional-pragmatischen Grundkompetenzen (Fragen 1a, 1b und 1c) mit Literalität als unabhängige Variable, aber ohne Kontrollvariablen dar. Es wird deutlich, dass Erwachsene mit einer Lese- und Schreibkompetenz auf den Alpha-Levels 1-3 im Durchschnitt mit einer signifikant niedrigeren Wahrscheinlichkeit angeben, die erfragten politikbezogenen Handlungen ohne Schwierigkeiten, bzw. nur mit gewissen Schwierigkeiten durchführen zu können. Im Modell ohne Kontrollvariablen gibt es also einen positiven Zusammenhang zwischen Literalität und selbsteingeschätzter Politikkompetenz.

In Tab. 2 werden zu den Modellen nun die Kontrollvariablen hinzugefügt. Als Hauptergebnis lässt sich festhalten, dass Literalität weiterhin einen positiven und signifikanten Effekt auf die selbsteingeschätzten politikbezogenen Grundkompetenzen hat. Bei allen drei Fragen liegt der Koeffizient für die Gruppe der gering literalisierten Erwachsenen unter eins. Dies bedeutet, dass gering literalisierte Personen mit einer höheren Wahrscheinlichkeit eine niedrigere Grundkompetenz in den hier untersuchten Bereichen angeben, selbst wenn auf weitere sozioökonomische Variablen hin kontrolliert wird.

Bei den Kontrollvariablen zeigt sich ein differenzierteres Bild. Das Geschlecht weist bei zwei Modellen (1b und 1c) einen signifikanten Effekt auf: Männer schätzen ihre politikbezogenen Grundkompetenzen höher ein als Frauen. Deutlich ist

Tab. 1 Ergebnisse der logistischen Regressionsanalyse der funktional-pragmatischen politikbezogenen Grundkompetenzen ohne Kontrollvariablen

\begin{tabular}{llll}
\hline & $\begin{array}{l}\text { (1a) Amt/Behörde (KI } \\
95 \%)\end{array}$ & $\begin{array}{l}(1 \mathrm{~b}) \text { pol. Frage (KI } \\
95 \%)\end{array}$ & $\begin{array}{l}\text { (1c) pol. Gespräch (KI } \\
95 \%)\end{array}$ \\
\hline $\begin{array}{l}\text { Literalität (Referenz: } \\
\text { Alpha-Level 4 und darüber) }\end{array}$ & \\
$\begin{array}{l}\text { McFaddens Pseu- } \\
\text { do } R^{2}\end{array}$ & 0,09 & $0,22(0,16-0,29)^{* * * *}$ & $0,23(0,18-0,31)^{* * *}$ \\
$N$ & 6725 & 0,04 & 0,04 \\
\hline
\end{tabular}

Koeffizienten als odds ratio

Konfidenzintervall $(95 \%)$ in Klammern

$* p<0,05 ; * * p<0,01 ; * * * p<0,001 ; n s$ nicht signifikant 
Tab. 2 Ergebnisse der logistischen Regressionsanalyse der funktional-pragmatischen politikbezogenen Grundkompetenzen mit Kontrollvariablen

\begin{tabular}{|c|c|c|c|}
\hline & $\begin{array}{l}\text { (1a) Amt/Behörde (KI } \\
95 \%)\end{array}$ & $\begin{array}{l}\text { (1b) pol. Frage (KI } \\
95 \%)\end{array}$ & $\begin{array}{l}\text { (1c) pol. Gespräch (KI } \\
95 \%)\end{array}$ \\
\hline \multicolumn{4}{|c|}{ Literalität (Referenz: Alpha-Level 4 und darüber) } \\
\hline Alpha-Levels 1-3 & $0,36(0,23-0,57)^{* * *}$ & $0,46(0,62-0,67)^{* * *}$ & $0,51(0,35-0,74)^{* * * *}$ \\
\hline \multicolumn{4}{|c|}{ Geschlecht (Referenz: weiblich) } \\
\hline Männlich & $1,31(0,97-1,77)^{\mathrm{ns}}$ & $2,09(1,64-2,67) * * *$ & $2,46(2,01-3,02) * * *$ \\
\hline \multicolumn{4}{|c|}{ Alter (Referenz: 55-64 Jahre) } \\
\hline 18-24 Jahre & $0,33(0,19-0,60) * * *$ & $0,27(0,17-0,43)^{* * *}$ & $0,25(0,17-0,37)^{* * * *}$ \\
\hline 25-34 Jahre & $0,42(0,27-0,65)^{* * * *}$ & $0,45(0,32-0,64)^{* * *}$ & $0,50(0,37-0,67)^{* * * *}$ \\
\hline 35-44 Jahre & $0,51(0,32-0,79)^{* * *}$ & $0,60(0,42-0,86)^{* * *}$ & $0,54(0,40-0,72)^{* * * *}$ \\
\hline 45-54 Jahre & $0,90(0,58-1,41)^{\mathrm{ns}}$ & $0,87(0,63-1,19)^{\mathrm{ns}}$ & $0,89(0,67-1,13)^{\mathrm{ns}}$ \\
\hline \multicolumn{4}{|c|}{ Schulabschluss (Referenz: hoher Schulabschluss) } \\
\hline Kein & $0,32(0,19-0,56) * * *$ & $0,31(0,19-0,52)^{* * * *}$ & $0,23(0,14-0,37) * * *$ \\
\hline Niedrig & $0,42(0,28-0,64)^{* * *}$ & $0,30(0,21-0,43)^{* * *}$ & $0,30(0,23-0,40)^{* * * *}$ \\
\hline Mittel & $0,90(0,61-1,32)^{\mathrm{ns}}$ & $0,50(0,56-1,15)^{\mathrm{ns}}$ & $0,51(0,40-0,66)^{* * *}$ \\
\hline \multicolumn{4}{|c|}{ Höchster Schulabschluss der Eltern (Referenz: hoher Schulabschluss) } \\
\hline Kein & $0,30(0,15-0,61)^{* * *}$ & $0,33(0,17-0,64) * * *$ & $0,48(0,26-0,91)^{*}$ \\
\hline Niedrig & $0,79(0,52-1,20)^{\mathrm{ns}}$ & $0,67(0,47-0,96)^{*}$ & $0,53(0,40-0,72)^{* * *}$ \\
\hline Mittel & $0,78(0,50-1,23)^{\mathrm{ns}}$ & $0,80(0,56-1,15)^{\mathrm{ns}}$ & $0,57(0,41-0,77)^{* * *}$ \\
\hline \multicolumn{4}{|c|}{ Erwerbsstatus (Referenz: erwerbstätig) } \\
\hline Erwerbslos & $0,63(0,39-1,01)^{\mathrm{ns}}$ & $0,71(0,48-1,06)^{\mathrm{ns}}$ & $0,66(0,46-0,94)^{*}$ \\
\hline Elternzeit & $1,99(0,61-6,45)^{\mathrm{ns}}$ & $1,07(0,59-1,96)^{\mathrm{ns}}$ & $1,16(0,65-2,07)^{\mathrm{ns}}$ \\
\hline In Ausbildung & $0,85(0,45-1,61)^{\mathrm{ns}}$ & $1,33(0,82-2,18)^{\mathrm{ns}}$ & $1,63(1,04-2,6)^{*}$ \\
\hline Im Ruhestand & $0,97(0,53-1,78)^{\mathrm{ns}}$ & $1,08(0,72-1,63)^{\mathrm{ns}}$ & $1,18(0,83-1,67)^{\mathrm{ns}}$ \\
\hline Sonstiger & $0,74(0,46-1,18)^{\mathrm{ns}}$ & $0,67(0,47-0,94)^{*}$ & $0,69(0,52-0,93) *$ \\
\hline \multicolumn{4}{|c|}{ Auskommen mit dem Haushalteinkommen (Referenz: gut/eher gut) } \\
\hline Schlecht/eher schlecht & $0,61(0,41-0,89)^{* * *}$ & $0,66(0,50-0,89)^{* * *}$ & $0,58(0,45-0,75)^{* * *}$ \\
\hline \multicolumn{4}{|c|}{ Herkunftssprache (Referenz: deutsche Herkunftssprache) } \\
\hline $\begin{array}{l}\text { Andere Herkunfts- } \\
\text { sprache }\end{array}$ & $0,65(0,36-1,20)^{\mathrm{ns}}$ & $0,48(0,29-0,78)^{* *}$ & $0,45(0,29-0,70)^{* * *}$ \\
\hline \multicolumn{4}{|c|}{ Staatsangehörigkeit (Referenz: deutsche Staatsangehörigkeit) } \\
\hline $\begin{array}{l}\text { Andere Staatsangehö- } \\
\text { rigkeit }\end{array}$ & $0,76(0,41-1,43)^{\mathrm{ns}}$ & $0,99(0,89-1,68)^{\mathrm{ns}}$ & $0,76(0,48-1,20)^{\mathrm{ns}}$ \\
\hline McFaddens Pseudo $R^{2}$ & 0,16 & 0,14 & 0,16 \\
\hline$N$ & 6510 & 6485 & 6444 \\
\hline
\end{tabular}

Koeffizienten als odds ratio

Konfidenzintervall $(95 \%)$ in Klammern

$* p<0,05 ; * * p<0,01 ; * * * p<0,001 ; n s$ nicht signifikant

das Ergebnis auch bei den Altersgruppen: Die beiden ältesten Gruppen im Sample (45-64 Jahre) geben im Vergleich zu den jüngeren Gruppen (18-44 Jahre) bei allen drei Fragen mit größerer Wahrscheinlichkeit eine höhere politikbezogene Kompetenz an.

Befragte, die keinen oder einen niedrigen Schulabschluss besitzen, weisen bei allen drei Fragen eine niedrigere politikbezogene Kompetenz auf. Bei Befragten 
mit einem mittleren Abschluss ist der Koeffizient nur bei der Frage (1c) nach der kompetenten Teilnahme an einem Gespräch über politische Fragen signifikant. Diese Kompetenz hat sich auch in der deskriptiven Untersuchung als eher schwierig herausgestellt (Dutz und Grotlüschen 2020, S. 312). Ein ähnliches Ergebnis zeigt sich beim höchsten Schulabschluss der Eltern: Der Schulabschluss der Eltern ist vor allem bei dieser Frage (1c) von Bedeutung.

Dagegen ist der Erwerbsstatus für die selbsteingeschätzte politikbezogene Grundkompetenz von eher untergeordneter Bedeutung. Wiederum bei der Frage nach der Teilnahme an einem Gespräch zu politischen Themen zeigt sich ein signifikanter Effekt der Kategorie ,erwerbslos“. Es zeigt sich jedoch, dass das Haushaltseinkommen einen signifikanten Effekt aufweist. Befragte, die angeben, dass sie mit dem Haushaltseinkommen schlecht bzw. eher schlecht zurechtkommen, besitzen eine signifikant höhere Wahrscheinlichkeit, ihre Kompetenz bei den drei Fragen geringer einzuschätzen. Hier scheint also das Zurechtkommen mit dem Haushaltseinkommen wichtiger zu sein als der individuelle Erwerbsstatus.

In Bezug auf die Herkunftssprache zeigt sich bei den Fragen (1b) und (1c) ein signifikanter negativer Einfluss, wenn die Befragten die deutsche Sprache nicht in ihrer Kindheit erlernt haben. Dieser Effekt ist vorhanden, obwohl die getesteten schriftsprachlichen Deutschkenntnisse in der Regression ebenfalls enthalten sind. Der Einfluss einer nicht-deutschen Staatsangehörigkeit ist dagegen nicht signifikant.

Zusammenfassend lässt sich für den Bereich der funktional-pragmatischen politikbezogenen Grundkompetenzen feststellen, dass gering literalisierte Erwachsene mit höherer Wahrscheinlichkeit eine niedrigere selbsteingeschätzte Grundkompetenz besitzen. Dieser Zusammenhang bleibt auch bestehen, wenn dem Regressionsmodell eine Reihe von Kontrollvariablen hinzugefügt wurde.

\subsection{Kritisch-hinterfragende Grundkompetenzen}

Bei der Regressionsanalyse ohne Kontrollvariablen zeigt sich hinsichtlich der kritisch-hinterfragenden Kompetenzen ein ähnliches Bild wie bei den funktional-pragmatischen Kompetenzen (s. Tab. 3). Gering literalisierte Erwachsene geben mit einer niedrigeren Wahrscheinlichkeit an, dass sie die erfragten Sachverhalte einfach bzw. eher einfach beurteilen können. Die Ergebnisse deuten also auch hier auf einen positiven Zusammenhang zwischen Literalität und selbsteingeschätzter Politikkompetenz hin.

In Tab. 4 finden sich die Ergebnisse der Regressionen der kritisch-hinterfragenden politikbezogenen Grundkompetenzen mit den Kontrollvariablen. Im Vergleich zu den Modellen der funktional-pragmatischen Kompetenzen zeigt sich zunächst eine durchgehend geringere Anpassungsgüte (McFaddens Pseudo R²) der gewählten Regressionsmodelle sowie eine vergleichsweise große Anzahl nicht signifikanter Koeffizienten. Eine Erklärung für dieses Verhalten könnte sein, dass ein hoher Anteil der Befragten auf die Fragen hinsichtlich kritisch-hinterfragender Grundkompetenzen mit ,weiß nicht“ antwortete, wie bereits oben erwähnt. Dies erschwert die Interpretation der Ergebnisse.

Betrachtet man die Ergebnisse der Regressionsanalysen hinsichtlich des Effekts der Literalität auf die kritisch-hinterfragenden Grundkompetenzen, so bestätigt sich 
Tab. 3 Ergebnisse der logistischen Regressionsanalyse der kritisch-hinterfragenden Grundkompetenzen ohne Kontrollvariablen

\begin{tabular}{llll}
\hline & $\begin{array}{l}\text { (2a) Unterschriften (KI } \\
95 \%)\end{array}$ & $\begin{array}{l}(2 \mathrm{~b}) \text { Demonstrationen }(\mathrm{KI} \\
95 \%)\end{array}$ & $\begin{array}{l}\text { (2c) Partei (KI } \\
95 \%)\end{array}$ \\
\hline $\begin{array}{l}\text { Literalität (Referenz: } \\
\text { Alpha-Level 4 und darüber) }\end{array}$ & & 0,28 \\
Alpha-Lels 1-3 & $0,18(0,14-0,24)^{* * *}$ & $0,30(0,23-0,40)^{* * *}$ & $(0,21-0,37)^{* * * *}$ \\
$\begin{array}{l}\text { McFaddens Pseu- } \\
\text { do } R^{2}\end{array}$ & 0,06 & 0,04 & 0,04 \\
$N$ & 6232 & 6321 & 6138 \\
\hline
\end{tabular}

Koeffizienten als odds ratio

Konfidenzintervall $(95 \%)$ in Klammern

$* p<0,05 ; * * p<0,01 ; * * * p<0,001 ; n s$ nicht signifikant

die Hypothese auch hier: Erwachsene mit literalen Fähigkeiten auf dem AlphaLevels 1-3 besitzen eine größere Wahrscheinlichkeit, ihre kritisch-hinterfragenden Grundkompetenzen im Mittel niedriger einzuschätzen als höher literalisierte Erwachsene. Dieser Befund ist eindeutig und die Koeffizienten durchgehend höchst signifikant.

Mit Blick auf das Geschlecht ist auch hier festzuhalten, dass Männer ihre politikbezogene Kompetenz höher einschätzen als Frauen. Bei der Betrachtung der Altersgruppen ist der Effekt nicht so eindeutig: In der Tendenz scheinen ältere Erwachsene ihre Fähigkeiten zwar als höher einzuschätzen. Gerade bei der Frage nach der Beurteilung der Teilnahme an einer Unterschriftenliste (2a) sind kaum Unterschiede zwischen den Altersgruppen zu beobachten; lediglich die jüngste Altersgruppe (18-24 Jahre) besitzt eine signifikant höhere Wahrscheinlichkeit, die eigene Kompetenz im Vergleich zur Referenzgruppe niedriger einzuschätzen.

Bei der Betrachtung des Schulabschlusses zeigt sich ein starker Effekt bei allen drei in diesem Kompetenzbereich erhobenen Fragen. Auch hier zeigt sich also die wichtige Rolle des eigenen Schulabschlusses bei der Beurteilung der eigenen kritisch-hinterfragenden Kompetenz.

Kaum eine Rolle spielt hingegen der Bildungshintergrund der Eltern. Die Koeffizienten gehen zwar in eine ähnliche Richtung wie beim eigenen Schulabschluss, sind aber nicht signifikant. Dem eigenen Schulerleben kann hier also eine wichtigere Funktion zugeschrieben werden als der familiären Herkunft.

Der Erwerbstatus spielt auch in diesem Kompetenzbereich zur Erklärung der Kompetenz keine wichtige Rolle. Eine Ausnahme ist die Frage danach, wie einfach/ schwierig es zu beurteilen sei, ob eine bestimmte politische Partei das vertritt, was man wichtig findet. Hier schätzen im Schnitt eher weniger erwerbslose Befragte ihre Kompetenz als eher hoch ein. Mit dem Haushaltseinkommen schlecht bzw. eher schlecht zurechtzukommen, ist jedoch bei zwei Fragen ( $2 \mathrm{a}$ und $2 \mathrm{~b}$ ) ein signifikanter Indikator für eine niedrige selbsteingeschätzte Kompetenz.

In Bezug auf die Herkunftssprache zeigt sich bei allen drei Fragen ein signifikanter negativer Einfluss, wenn die Herkunftssprache nicht Deutsch ist. Wie bei den funktional-pragmatischen politikbezogenen Grundkompetenzen ist dieser Effekt vorhanden, obwohl die getesteten schriftsprachlichen Deutschkenntnisse in der Re- 
Tab. 4 Ergebnisse der logistischen Regressionsanalyse der kritisch-hinterfragenden politikbezogenen Grundkompetenzen mit Kontrollvariablen

\begin{tabular}{|c|c|c|c|}
\hline & $\begin{array}{l}\text { (2a) Unterschriften (KI } \\
95 \%)\end{array}$ & $\begin{array}{l}\text { (2b) Demonstrationen } \\
\text { (KI 95\%) }\end{array}$ & $\begin{array}{l}\text { (2c) Partei (KI } \\
95 \%)\end{array}$ \\
\hline \multicolumn{4}{|c|}{ Literalität (Referenz: Alpha-Level 4 und darüber) } \\
\hline Alpha-Levels 1-3 & $0,44(0,31-0,62)^{* * *}$ & $0,54(0,39-0,75)^{* * *}$ & $\begin{array}{l}0,57 \\
(0,41-0,79)^{* * * *}\end{array}$ \\
\hline \multicolumn{4}{|c|}{ Geschlecht (Referenz: weiblich) } \\
\hline Männlich & $1,15(0,98-1,38)^{\mathrm{ns}}$ & $1,47(1,26-1,71)^{* * *}$ & $1,23(1,04-1,46)^{*}$ \\
\hline \multicolumn{4}{|c|}{ Alter (Referenz: 55-64 Jahre) } \\
\hline 18-24 Jahre & $0,65(0,46-0,95)^{*}$ & $0,52(0,37-0,73)^{* * *}$ & $\begin{array}{l}0,59 \\
(0,41-0,86)^{* *}\end{array}$ \\
\hline 25-34 Jahre & $0,89(0,66-1,20)^{\mathrm{ns}}$ & $0,63(0,50-0,81)^{* * *}$ & $0,73(0,55-0,96)^{*}$ \\
\hline 35-44 Jahre & $0,97(0,73-1,29)^{\mathrm{ns}}$ & $0,84(0,67-1,07)^{\mathrm{ns}}$ & $0,80(0,62-1,05)^{\mathrm{ns}}$ \\
\hline 45-54 Jahre & $0,84(0,65-1,09)^{\mathrm{ns}}$ & $0,69(0,56-0,85)^{* * *}$ & $\begin{array}{l}0,72 \\
(0,56-0,91)^{* *}\end{array}$ \\
\hline \multicolumn{4}{|c|}{ Schulabschluss (Referenz: hoher Schulabschluss) } \\
\hline Kein & $0,39(0,24-0,63)^{* * *}$ & $0,37(0,24-0,57)^{* * *}$ & $\begin{array}{l}0,46 \\
(0,29-0,72)^{* * * *}\end{array}$ \\
\hline Niedrig & $0,46(0,35-0,60) * * *$ & $0,52(0,41-0,65)^{* * *}$ & $\begin{array}{l}0,43 \\
(0,34-0,56)^{* * * *}\end{array}$ \\
\hline Mittel & $0,67(0,53-0,86)^{* *}$ & $0,77(0,64-0,93)^{* *}$ & $\begin{array}{l}0,73 \\
(0,59-0,91)^{* *}\end{array}$ \\
\hline \multicolumn{4}{|c|}{ Höchster Schulabschluss der Eltern (Referenz: hoher Schulabschluss) } \\
\hline Kein & $0,71(0,37-1,35)^{\mathrm{ns}}$ & $0,43(0,23-0,80)^{* *}$ & $0,56(0,29-1,10)^{\mathrm{ns}}$ \\
\hline Niedrig & $0,80(0,61-1,06)^{\mathrm{ns}}$ & $0,86(0,69-1,07)^{\mathrm{ns}}$ & $0,79(0,61-1,03)^{\mathrm{ns}}$ \\
\hline Mittel & $1,07(0,81-1,41)^{\mathrm{ns}}$ & $0,90(0,73-1,12)^{\mathrm{ns}}$ & $0,99(0,77-1,29)^{\mathrm{ns}}$ \\
\hline \multicolumn{4}{|c|}{ Erwerbsstatus (Referenz: erwerbstätig) } \\
\hline Erwerbslos & $0,84(0,59-1,19)^{\mathrm{ns}}$ & $1,03(0,75-1,42)^{\mathrm{ns}}$ & $0,68(0,49-0,95)^{*}$ \\
\hline Elternzeit & $1,00(0,51-1,95)^{\mathrm{ns}}$ & $1,01(0,67-1,53)^{\mathrm{ns}}$ & $1,12(0,67-1,87)^{\mathrm{ns}}$ \\
\hline In Ausbildung & $0,95(0,61-1,49)^{\mathrm{ns}}$ & $0,85(0,61-1,18)^{\mathrm{ns}}$ & $0,88(0,59-1,30)^{\mathrm{ns}}$ \\
\hline Im Ruhestand & $0,88(0,64-1,21)^{\mathrm{ns}}$ & $1,12(0,87-1,45)^{\mathrm{ns}}$ & $0,79(0,68-1,21)^{\mathrm{ns}}$ \\
\hline Sonstiger & $0,85(0,63-1,15)^{\mathrm{ns}}$ & $1,10(0,85-1,39)^{\mathrm{ns}}$ & $0,90(0,68-1,22)^{\mathrm{ns}}$ \\
\hline \multicolumn{4}{|c|}{ Auskommen mit dem Haushalteinkommen (Referenz: gut/eher gut) } \\
\hline Schlecht/eher schlecht & $0,69(0,53-0,89)^{*}$ & $0,77(0,62-0,97)^{*}$ & $0,96(0,74-1,25)^{\mathrm{ns}}$ \\
\hline \multicolumn{4}{|c|}{ Herkunftssprache (Referenz: deutsche Herkunftssprache) } \\
\hline $\begin{array}{l}\text { Andere Herkunfts- } \\
\text { sprache }\end{array}$ & $0,39(0,26-0,59)^{* * *}$ & $0,58(0,39-0,87)^{* *}$ & $\begin{array}{l}0,50 \\
(0,33-0,75)^{* * * *}\end{array}$ \\
\hline \multicolumn{4}{|c|}{ Staatsangehörigkeit (Referenz: deutsche Staatsangehörigkeit) } \\
\hline $\begin{array}{l}\text { Andere Staatsangehö- } \\
\text { rigkeit }\end{array}$ & $0,65(0,42-1,01)^{\mathrm{ns}}$ & $0,87(0,57-1,32)^{\mathrm{ns}}$ & $0,84(0,55-1,28)^{\mathrm{ns}}$ \\
\hline McFaddens Pseudo $R^{2}$ & 0,11 & 0,07 & 0,07 \\
\hline$N$ & 6030 & 6122 & 5940 \\
\hline
\end{tabular}

Koeffizienten als odds ratio

Konfidenzintervall $(95 \%)$ in Klammern

$* p<0,05 ; * * p<0,01 ; * * * p<0,001 ; n s$ nicht signifikant 
gression ebenfalls enthalten sind. Keinen signifikanten Einfluss zeigt auch hier eine nicht-deutsche Staatsangehörigkeit.

\section{Limitationen}

Das Sample ist durch die Anlage von LEO 2018 auf die deutschsprachige Wohnbevölkerung in Privathaushalten im Alter von 18 bis 64 Jahren begrenzt. Dies schließt insbesondere jüngere und ältere Menschen aus, ebenso Menschen, die nicht ausreichend gut deutsch sprechen, um der Befragung zu folgen. Ebenso sind Personen, die in institutionellen Wohnformen, wie Pflegeheimen, Flüchtlingsunterkünften oder Justizvollzugsanstalten leben, nicht in der Stichprobe enthalten. Es kann vermutet werden, dass diese Gruppen strukturell häufiger von politischer Teilhabe ausgeschlossen sind und die hier präsentierten Ergebnisse deshalb die gesamtgesellschaftlichen Ausschlüsse eher unterschätzen.

Studien zeigen, dass selbsteingeschätzte Kompetenzen häufig nicht den getesteten Kompetenzen entsprechen und bestimmte Gruppen die eigenen Fähigkeiten systematisch über- bzw. unterschätzen (eine aktuelle Studie mit ausführlichem Literaturüberblick findet sich bei Edele et al. 2015). Die hier durchgeführte Analyse ist gerade an dem subjektiven Blick auf die eigenen Kompetenzen interessiert. Es sei dennoch darauf hingewiesen, dass die untersuchten politikbezogenen Grundkompetenzen auf Selbstauskünften und nicht auf einem Test basieren und entsprechend interpretiert werden sollten.

\section{Diskussion}

Ziel dieses Beitrags war es, den Zusammenhang zwischen Literalität und selbsteingeschätzten politikbezogenen Grundkompetenzen zu untersuchen. Im Ergebnis lässt sich festhalten, dass die Analysen einen eindeutigen Zusammenhang zwischen getesteter Literalität und selbsteingeschätzten politikbezogenen Grundkompetenzen aufzeigt. Die Gruppe der gering literalisierten Erwachsenen zeigt eine erhöhte Wahrscheinlichkeit, die eigenen politikbezogenen Grundkompetenzen als eher niedrig einzuschätzen. Dies gilt sowohl für die funktional-pragmatischen als auch die kritisch-hinterfragenden Kompetenzen. Die Hypothese konnte somit bestätigt werden. Während sich das Geschlecht, das Alter, der Schulabschluss, das Haushaltseinkommen und die Herkunftssprache als gute Prädikatoren erwiesen haben, war dies beim höchsten Schulabschluss der Eltern, dem Erwerbsstatus und der Staatsangehörigkeit nicht der Fall. Außerdem zeigen die Modelle der kritisch-hinterfragenden Kompetenzen eine schlechtere Modellpassung. Dies deutet darauf hin, dass die hier verwendeten sozio-ökonomischen Faktoren nur zum Teil geeignet sind, die kritischhinterfragenden Grundkompetenzen im Modell vorherzusagen.

Für die Erwachsenenbildung bedeutet dies eine Bestätigung der These, dass gering literalisierte Erwachsene über niedrige selbsteingeschätzte politikbezogene Kompetenzen verfügen. Damit geht nicht zwingend einher, dass diese tatsächlich über eine geringe Politikkompetenz verfügen - auch wenn subjektive und getestete 
Fähigkeiten häufig miteinander korrelieren. Es besteht aber die Gefahr, dass - wie bei Kursteilnehmenden und bei anderen gesellschaftlichen Gruppen beobachtet und oben dargestellt - ein Selbstausschluss vom politischen Feld stattfindet. Im Kontext der politischen Bildung interpretiert Bremer (2010) dies als ,,vorweggenommene Fremdausschließung“ (Bremer 2010, S. 186). Butterwegge (2015) argumentiert ähnlich und kritisiert, dass die Belange bestimmter Gruppen von der Politik wegen ihrer vermeintlichen Politikferne nicht beachtet würden und sich so ein Gefühl der Einflusslosigkeit einstellen könne - letztlich eine Art selbsterfüllender Prophezeiung.

Gemeinsam mit einem niedrigen Selbstwirksamkeitsgefühl, geringem sozialen Vertrauen und einem Gefühl, die aktuellen politischen Probleme nicht durchschauen oder darauf Einfluss nehmen zu können, besteht die Gefahr der Entsolidarisierung mit der Gesellschaft und der Hinwendung zu populistischen und rechtsextremen Parteien (vgl. Grotlüschen 2016) oder ein genereller Verzicht auf die politische Beteiligung (z. B. bei Wahlen). Der Teilhabeausschluss ist deutlich und erfordert ein Handeln der Alphabetisierung und Grundbildung, wie auch die insgesamt zunehmende Diskussion um die politische Grundbildung in den letzten Jahren zeigt. Entsprechende Angebote der politischen Bildung können solchen Entwicklungen entgegenwirken (Martin und Reichart 2020) und sollten daher nicht nur politisches Fachwissen, sondern auch die Legitimität des eigenen politischen Handelns vermitteln, unabhängig von den individuellen literalen oder politischen Kompetenzen. Eine hier nicht betrachtete Frage ist, wie die selbsteingeschätzten politikbezogenen Grundkompetenzen die politische Partizipation beeinflussen und wo genau die Ursache für die im Schnitt geringere politische Partizipation gering literalisierter Erwachsener (Dutz und Grotlüschen 2020) liegt.

Die in diesem Beitrag nur angerissenen Ergebnisse von LEO 2018 und Erfahrungen aus der Praxis zeigen auch, dass die voneinander getrennte Behandlung der verschiedenen Grundbildungsbereiche im Alltag häufig lebensfern und nicht zielführend ist. Eine an den Prinzipien der politischen Erwachsenenbildung ausgerichtete politische Grundbildung, die sich ,,an den Lerninteressen und Lernbedürfnissen und Lernvoraussetzungen der Teilnehmenden orientiert“" (Zeuner 2017, S. 43), kann dies leisten. So erfordert etwa das Erkennen von Fake News neben Politikkompetenz auch digitale Medienkompetenz, ebenso die Nutzung moderner Partizipationsformen im Internet. Die gewissenhafte Beurteilung verpflichtender Coronatests nach dem Sommerurlaub verlangt neben einer politischen Einordnung auch gesundheitsbezogene Kompetenzen. Dies ist umso relevanter, da gering literalisierte Erwachsene sich auch in diesen Kompetenzbereichen eher unterdurchschnittliche Fähigkeiten bescheinigen (Buddeberg und Grotlüschen 2020; Heilmann 2020).

Die Grundbildungspraxis und die Forschung zum Thema Grundbildung sind in Deutschland gegenwärtig stark in die Nationale Dekade für Alphabetisierung und Grundbildung (BMBF und Kultusministerkonferenz 2016) eingebunden. Damit ist sie noch bis zum Jahr 2026 institutionell auf Bundes- und Länderebene und zivilgesellschaftlich verankert und mittelfristig abgesichert. Befunde $\mathrm{zu}$ politischen Grundkompetenzen im Zusammenhang mit vulnerablen Gruppen, zu denen gering literalisierte Erwachsene fraglos zu zählen sind, können den Diskurs bereichern. Es zeigt sich deutlich, dass Grundbildung - wie häufig formelhaft beschworen - in der 
Tat mehr ist als Lesen und Schreiben, und dass politische und populismuskritische Bildung einen festen Platz im Grundbildungskanon beanspruchen darf.

Open Access Dieser Artikel wird unter der Creative Commons Namensnennung 4.0 International Lizenz veröffentlicht, welche die Nutzung, Vervielfältigung, Bearbeitung, Verbreitung und Wiedergabe in jeglichem Medium und Format erlaubt, sofern Sie den/die ursprünglichen Autor(en) und die Quelle ordnungsgemäß nennen, einen Link zur Creative Commons Lizenz beifügen und angeben, ob Änderungen vorgenommen wurden.

Die in diesem Artikel enthaltenen Bilder und sonstiges Drittmaterial unterliegen ebenfalls der genannten Creative Commons Lizenz, sofern sich aus der Abbildungslegende nichts anderes ergibt. Sofern das betreffende Material nicht unter der genannten Creative Commons Lizenz steht und die betreffende Handlung nicht nach gesetzlichen Vorschriften erlaubt ist, ist für die oben aufgeführten Weiterverwendungen des Materials die Einwilligung des jeweiligen Rechteinhabers einzuholen.

Weitere Details zur Lizenz entnehmen Sie bitte der Lizenzinformation auf http://creativecommons.org/ licenses/by/4.0/deed.de.

\section{Literatur}

Best, H., \& Wolf, C. (2010). Logistische Regression. In C. Wolf \& H. Best (Hrsg.), Handbuch der sozialwissenschaftlichen Datenanalyse (S. 827-854). Wiesbaden: Springer VS.

BMBF \& Kultusministerkonferenz (2016). Grundsatzpapier zur Nationalen Dekade für Alphabetisierung und Grundbildung 2016-2026. Den funktionalen Analphabetismus in Deutschland verringern und das Grundbildungsniveau erhöhen. https://www.alphadekade.de/files/01_Grundsatzpapier\%20zur $\%$ 20Nationalen \%20Dekade\%20Alphabetisierung\%20und\%20Grundbildung_final.pdf. Zugegriffen: 22. Okt. 2020

Bourdieu, P. (1987). Die feinen Unterschiede. Kritik der gesellschaftlichen Urteilskraft. Frankfurt a. M.: Suhrkamp.

Bourdieu, P. (2013). Das politische Feld. In F. Schultheis \& S. Egger (Hrsg.), Schriften 07: Politik. Schriften zur Politischen Ökonomie, (Bd. 2, S. 97-112). Frankfurt a. M.: Suhrkamp.

Bremer, H. (2010). Symbolische Macht und politisches Feld. Der Beitrag der Theorie Pierre Bourdieus für die politische Bildung. In B. Lösch \& A. Thimmel (Hrsg.), Kritische politische Bildung. Ein Handbuch (S. 181-192). Schwalbach: Wochenschau.

Bremer, H., \& Pape, N. (2017). Literalität und Partizipation als milieuspezifische soziale Praxis. In B. Menke \& W. Riekmann (Hrsg.), Politische Grundbildung. Inhalte - Zielgruppen - Herausforderungen (S. 56-73). Schwalbach: Wochenschau.

Buddeberg, K., \& Grotlüschen, A. (2020). Literalität, digitale Praktiken und Grundkompetenzen. In A. Grotlüschen \& K. Buddeberg (Hrsg.), LEO 2018 - Leben mit geringer Literalität (S. 197-225). Bielefeld: wbv Publikation.

Butterwegge, C. (2015). Hartz IV und die Folgen. Auf dem Weg in eine andere Republik? Weinheim: Beltz Juventa.

van Deth, J.W. (2009). Politische Partizipation. In V. Kaina \& A. Römmele (Hrsg.), Politische Soziologie. Ein Studienbuch (1. Aufl. S. 141-161). Wiesbaden: Springer VS.

van Deth, J.W. (2013a). Das schwierige Verhältnis zwischen Partizipation und Demokratie. Politische Bildung, 3, 9-21.

van Deth, J.W. (2013b). Sind Partizipierende die besseren Demokraten? In S. I. Keil \& S. I. Thaidigsmann (Hrsg.), Zivile Bürgergesellschaft und Demokratie (S. 35-52). Wiesbaden: Springer VS.

Duncker-Euringer, C. (2017). Was ist Grundbildung? In B. Menke \& W. Riekmann (Hrsg.), Politische Grundbildung. Inhalte - Zielgruppen - Herausforderungen (S. 13-33). Schwalbach: Wochenschau.

Dutz, G., \& Grotlüschen, A. (2020). Literalität, politikbezogene Praktiken und Grundkompetenzen. In A. Grotlüschen \& K. Buddeberg (Hrsg.), LEO 2018 - Leben mit geringer Literalität (S. 287-322). Bielefeld: wbv Publikation.

Dutz, G., \& Hartig, J. (2020). Skalierung und Verlinkung der LEO-Studie 2018. In A. Grotlüschen \& K. Buddeberg (Hrsg.), LEO 2018 - Leben mit geringer Literalität (S. 65-77). Bielefeld: wbv Publikation.

Dutz, G., \& Heilmann, L. (2020). Lesekompetenz und politische Partizipation: Empirische Befunde aus PIAAC-L. In O. Dörner, C. Iller, I. Schüßler, H. von Felde \& S. Lerch (Hrsg.), Erwachsenenbildung 
und Lernen in Zeiten von Globalisierung, Transformation und Entgrenzung (S. 193-205). Opladen: B. Budrich.

Edele, A., Seuring, J., Kristen, C., \& Stanat, P. (2015). Why bother with testing? The validity of immigrants' self-assessed language proficiency. Social science research. https://doi.org/10.1016/j. ssresearch.2014.12.017.

Fuchs, G. (2006). Politische Partizipation von Frauen in Deutschland. In B. Hoecker (Hrsg.), Politische Partizipation zwischen Konvention und Protest (S. 235-260). Opladen: B. Budrich.

Grimm, P., Keber, T.O., \& Zöllner, O. (Hrsg.). (2019). Digitale Ethik. Leben in vernetzten Welten. Kompaktwissen XL. Stuttgart: Reclam.

Grotlüschen, A. (2016). Politische Grundbildung - Theoretische und empirische Annäherungen. Zeitschrift für Weiterbildungsforschung, 2(39), 183-203. https://doi.org/10.1007/s40955-016-0063-z.

Grotlüschen, A., \& Buddeberg, K. (Hrsg.). (2020). LEO 2018 - Leben mit geringer Literalität. Bielefeld: wbv Publikation.

Grotlüschen, A., \& Riekmann, W. (Hrsg.). (2012). Funktionaler Analphabetismus in Deutschland. Ergebnisse der ersten leo.-Level-One Studie. Münster: Waxmann.

Grotlüschen, A., Buddeberg, K., Dutz, G., Heilmann, L., \& Stammer, C. (2020). Hauptergebnisse und Einordnung zur LEO-Studie 2018 - Leben mit geringer Literalität. In A. Grotlüschen \& K. Buddeberg (Hrsg.), LEO 2018 - Leben mit geringer Literalität (S. 13-64). Bielefeld: wbv Publikation.

Grotlüschen, A., Heinemann, A. M. B., \& Nienkemper, B. (2009). Die unterschätzte Macht legitimer Literalität. REPORT, 4(32), 55-67.

Grotlüschen, A., Mallows, D., Reder, S., \& Sabatini, J. (2016). Adults with low proficiency in literacy or numeracy. Paris: OECD Publishing.

Hartig, J., Frey, A., Nold, G., \& Klieme, E. (2012). An application of explanatory item response modeling for model-based proficiency scaling. Educational and Psychological Measurement, 4(72), 665-686. https://doi.org/10.1177/0013164411430707.

Heilmann, L. (2020). Literalität, gesundheitsbezogene Praktiken und Grundkompetenzen. In A. Grotlüschen \& K. Buddeberg (Hrsg.), LEO 2018 - Leben mit geringer Literalität (S. 255-285). Bielefeld: wbv Publikation.

Klieme, E., \& Leutner, D. (2006). Kompetenzmodelle zur Erfassung individueller Lernergebnisse und zur Bilanzierung von Bildungsprozessen. Zeitschrift für Pädagogik, 6(52), 876-903.

Koppel, I., \& Schieferdecker, R. (2019). Alphabetisierung als Voraussetzung gesellschaftlicher Teilhabe und Transformation? Systematische Betrachtungen zum Schriftspracherwerb. In G. Lang-Wojtasik (Hrsg.), Bildung für eine Welt in Transformation. Global Citizenship Education als Chance für die Weltgesellschaft (S. 161-175). Opladen: B. Budrich.

Korfkamp, J. (2016). Politische Grundbildung. In M. Löffler \& J. Korfkamp (Hrsg.), Handbuch zur Alphabetisierung und Grundbildung (S. 458-466). Münster: Waxmann.

Lumley, T. (2018a). Mitools. Tools for multiple imputation of missing data

Lumley, T. (2018b). Survey. Analysis of complex survey samples

Mania, E., \& Tröster, M. (2018). Inhaltsbereiche der Grundbildung: Stand und Herausforderungen. In Agentur für Erwachsenen- \& Weiterbildung (Hrsg.), Grundbildung lebensnah gestalten. Fallbeispiele aus den Regionalen Grundbildungszentren in Niedersachsen (S. 11-21). Bielefeld: wbv Publikation.

Martin, A., \& Reichart, E. (2020). Zum Einfluss der politischen Bildung an Volkshochschulen auf die Wahlbeteiligung. In J. Schrader, A. Ioannidou \& H.-P. Blossfeld (Hrsg.), Monetäre und nicht monetäre Erträge von Weiterbildung (S. 175-211). Wiesbaden: Springer VS.

Müller, H.-P. (2016). Pierre Bourdieu. Eine systematische Einführung. Frankfurt a. M.: Suhrkamp.

Negt, O. (1990). Überlegungen zur Kategorie ,Zusammenhang ‘ als einer gesellschaftlichen Schlüsselqualifikation. REPORT Literatur- und Forschungsreport Weiterbildung, 4(26), 11-19.

Pape, N. (2011). Politische Partizipation aus der Sicht funktionaler Analphabet/inn/en. Report-Zeitschrift für Weiterbildungsforschung, 3(34), 15-23. https://doi.org/10.3278/REP1103W015.

Verba, S., Schlozman, K. L., \& Brady, H.E. (1995). Voice and equality. Civic voluntarism in American politics. Cambridge, London: Harvard University Press.

Wiedemann, C. (2006). Politische Partizipation von Menschen mit Migrationshintergrund. In B. Hoecker (Hrsg.), Politische Partizipation zwischen Konvention und Protest (S. 261-286). Opladen: B. Budrich.

Zeuner, C. (2017). Was ist/was gehört zur politischen Grundbildung? In B. Menke \& W. Riekmann (Hrsg.), Politische Grundbildung. Inhalte - Zielgruppen - Herausforderungen (S. 34-55). Schwalbach: Wochenschau.

Publisher's Note Springer Nature remains neutral with regard to jurisdictional claims in published maps and institutional affiliations. 